\title{
Quantum Computing and Complexity in Art
}

Libby Heaney (artist, $\mathrm{PhD}$ quantum physicist, tutor on Information Experience Design at the Royal College of Art). Royal College of Art, Kensington Gore, London, SW7 2EU, UK. Email: libby.heaney@rca.ac.uk

\section{(C) ISAST}

Manuscript received 9 August 2016.

ABSTRACT: The author draws on her research experience in quantum computing to discuss the conception and form of an interactive installation CLOUD. CLOUD explores complexity in the post-digital by referencing the principles of quantum superposition, quantum entanglement and quantum measurement.

Understanding complexity is the most critical issue of our times. So often, intricately detailed concepts or narratives are reduced to simple natural versus artificial, or good versus evil tales and propagated by the (social) media. For instance, Adam Curtis explores the complexity in popular reductive narratives through his films Bitterlake [1] and Hypernormalization [2] and George Marshall explores narratives around climate change in his book Don't Even Think About It [3]. For all cultural practitioners it is important to research and experiment with new forms and techniques enabling people to understand, experience and interact with complexity on their own terms. I believe concepts from quantum computing will bring new and wider techniques into our toolbox for articulating complexity and I will use my post-digital installation CLOUD to unpack these issues throughout this paper.

Complexity is a generic feature of large systems [4] and thus occurs across disciplines from biology to finance. As such its definition is notoriously difficult to pin down - for instance one may talk about computational complexity (the difficultly of solving certain computational problems [5]) or the complexity of an artwork or jazz composition (as the depth to the piece [6]). Johnson [7] wrote about properties of classical complex systems in Emergence and artists, such as Heather Barnett, Keith Tyson, Jennifer Steinkamp [8, 9, 10], focus on investigating concepts from such systems and creating metaphorical connections.

It is easy to believe that systems cannot be more complex than biology or finance. However, complexity increases exponentially when zooming into the fundamental constituents of the universe. Quantum mechanics describes the universe at its most basic level and quantum systems are inherently complex [11]. Importantly, this complexity is different to that of classical (nonquantum) systems. For instance, the superposition principle enables a single quantum particle to span infinite dimensions [12] and entangled states of many particles increase the complexity further. Eisert has argued that most composite quantum systems are too complex (entangled) to perform computation [13]. Therefore we may ask whether referencing quantum effects through art and design practices would provide new methods for articulating complexity? I will argue in the affirmative, while noting some challenges along the way.

Firstly, it is important to question why quantum physics is relevant to our everyday experience, since the physics of the systems mentioned above, from the notes in a jazz composition to a 
biological cell, is classical. And while quantum physics is not usually applied to macroscopic systems, a recent cutting edge theory called quantum interactions [14] argues that quantum logic can be applied away from quantum physics' usual microscopic domain. That is, parts of the quantum mathematical framework appear in non-physical aspects of psychology, economics, cognition and computational linguistics. Quantum theory is thus related to how humans think and communicate and therefore a potential tool for the exploration of complexity through creative practices.

In fact, using quantum physics as a metaphor for complex systems is not new. Ascott has discussed it extensively in the context of telematic systems and meaning since the 1960s. More recently, Barad used Bohr's philosophy-physics to develop her theory of agential reality [15] and Plotnitsky explores relationships/disparities between Bohr's complementarity and poststructuralist theory, notably Derrida [16].

There are some contradictions in referencing quantum effects through creative practices, however, since genuine quantum effects cannot be represented in macroscopic, physical artworks as the Schroedinger's cat paradox highlights [supplementary 1]. However, I do not believe this is a problem. As James Elkins puts it when discussing quantum physics imagery [17], "there is an inherent, systemic contradiction in this practice: it says both "quantum phenomena need to be visualized [in order to teach it]" and "quantum phenomena aren't amenable to visualization". However quantum physics images are useful as "free versions of a rigorous practice, intended to be suggestive and approximate rather than dependable and exact. ... Even images regarded by members of the physics community as patently inadequate or misguided can also come to seem potentially helpful." So while CLOUD focuses on experience as well as representation, it could also be viewed as a sequence of images that renders quantum computing both "unpicturable and picturable", articulating complexity through traces of properties that are essentially inconceivable.

CLOUD

CLOUD is an installation that spans digital and physical. The physical consists of 54 independently rotating, octahedron 'pixels' made from paper, tessellated within in a handknotted, linen net that in turn is suspended by a scaffold frame (commissioned as part of a wider exhibition) (Fig. 1).

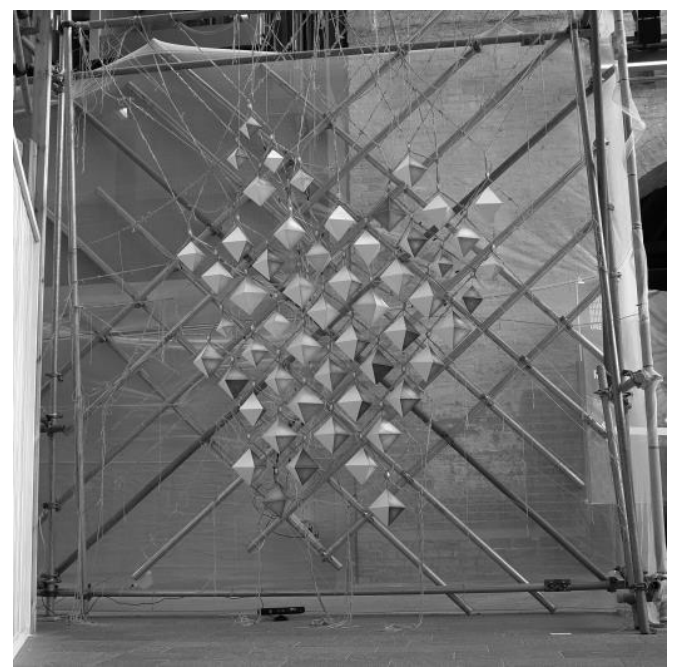

Fig. 1, CLOUD, Libby Heaney, 2015. (@ Libby Heaney 
Each pixel is shaded half with graphite and the other half is left as blank paper (Fig. 2). Behind are the electronics - namely a Servo motor for each pixel and $\sim 500 \mathrm{~m}$ of wiring. The orientations of the pixels are determined, in part, by data from a Microsoft Kinect depth camera, which senses the area in front of the piece, where a coded, invisible copy of the physical structure is digitally 'reflected' across the floor.

The presence of people in this space causes CLOUD to change its configuration. Before anyone enters the space, the pixels are in an ordered, low-entropy configuration: every single one is facing the same way (half-coloured and half-blank). Then, as participants walk around in the space, their paths are gradually mapped onto CLOUD through a random orientation of the corresponding pixels (Fig. 4). Each location in front of CLOUD has a one to one link with a pixel such that when a person steps into a location, the code controlling the installation randomly chooses to rotate the linked pixel left or right to reveal fully its shaded or blank side [S-2 - code] (Fig. 7). Overall, the installation thus evolves from an ordered to a random state, contingent on how people move in the space, with the pixels slowly re-ordering themselves after people have moved away from the piece for a certain amount of time.

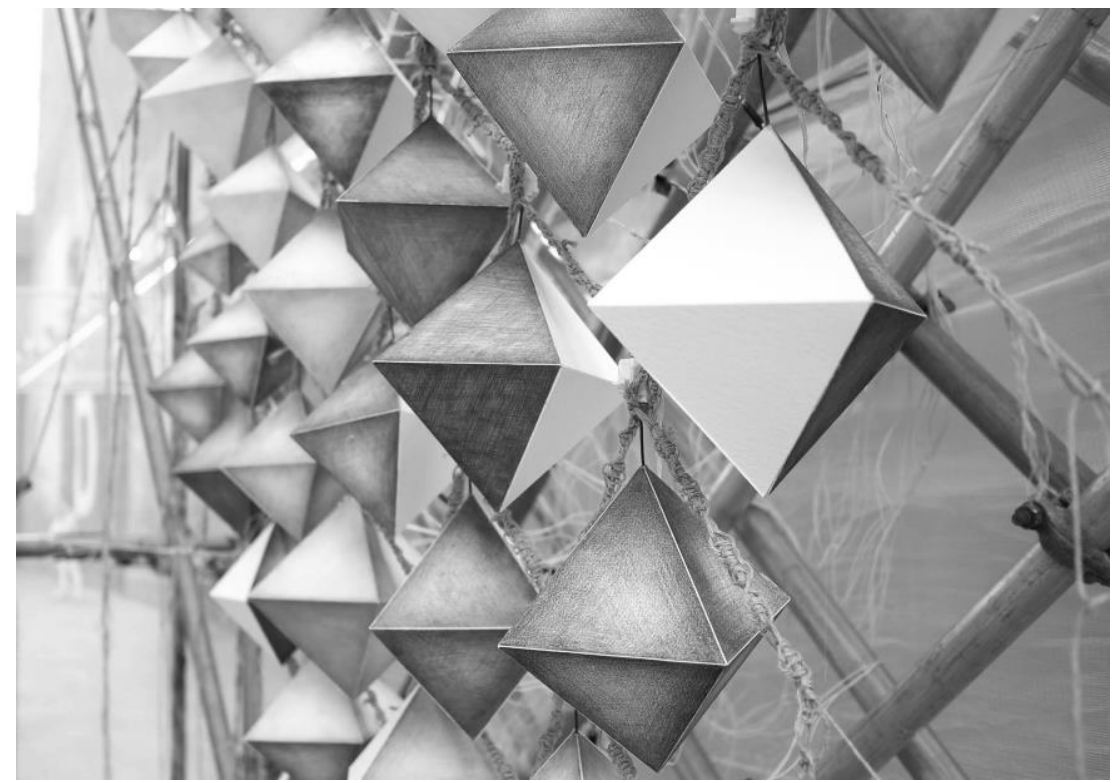

Fig. 2, close up of CLOUD showing graphite and white octahedrons, 2015. (@ Libby Heaney)

The functioning of CLOUD was inspired, in part, by three features of quantum information science [18]. Firstly, the superposition principle, which states that any quantum system can be in multiple states simultaneously. This could be a single massive particle such as a carbon C-60 'bucky ball' molecule passing through two spatially separated splits, for instance [19]. When applied to computation a bit then becomes a quantum bit (or qubit) and is no longer in either the zero or one state, but may be in both simultaneously. Superposition principle is somewhat like the famous gestalt image of two faces or a vase - depending on where in the image the observer looks they will see one or the other representation.

The second feature that informed this installation is quantum entanglement. This is the extension of quantum superposition for two or more quantum particles. In entangled states, the information 
about the properties of each particle (for instance their spin or position) is delocalised over all of the particles and the entire system is in a superposition of various configurations [20].

Entanglement is illustrated, again in a 'suggestive and approximate' way, by placing two Necker cubes next to each other (Fig. 3). The viewer sees either both cubes with one perspective or the other. The orientations of the two cubes are correlated - one never sees them with opposite orientations provided they are next to each other. Further they both exist in two states simultaneously.
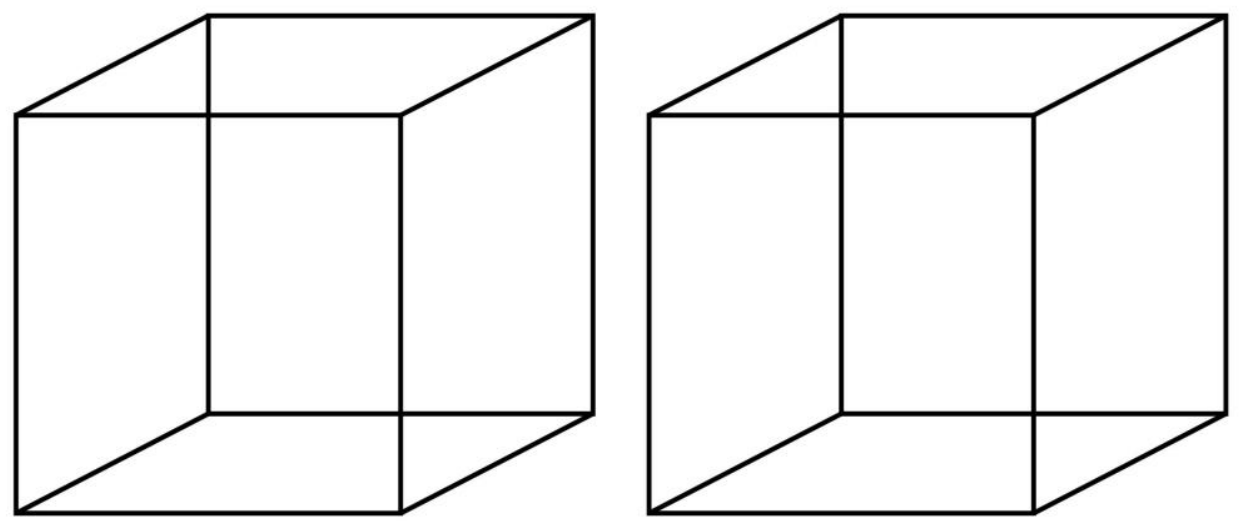

Fig. 3, two Necker cubes placed next to each other illustrate the correlations of entanglement.

The third property is quantum measurement. An act of observation irreversibly and ontologically changes the state of a quantum system, which 'decoheres' it into one classically observable state probabilistically. Here there are loose metaphorical connections to second order cybernetics and Bentham's panopticon model of a prison [supplementary 3], which will be explored in depth elsewhere.

These three concepts come together in quantum computing where certain algorithms process information more efficiently than classical computers. There are many proposed architectures for quantum computing. The scheme that inspired CLOUD is called measurement based quantum computation [21], where the utilization of entanglement and quantum measurement to drive computation can be seen most strikingly.

Measurement based quantum computation proceeds as follows. Before the computation takes place a large entangled state, called a cluster state, is created between hundreds of spatially localized qubits [22]. This forms a grid-like structure, in some sense, like hundreds of Necker cubes arranged in a square. For massive particles, qubits are logically represented by electron spin. The entanglement between neighbouring particles means that the information about the orientations of individual spins is nonlocally distributes over the entire system [23]. Once a cluster state is engineered, the computation proceeds via a sequence of different measurements that themselves encode the algorithm - on the individual qubits in the cluster state. These measurements destroy the spin-entanglement between the measured qubits and the rest of the lattice. The measured qubits are in random states, but the remaining parts of the cluster state yet to be measured - draws closer to the final solution of the computation. 


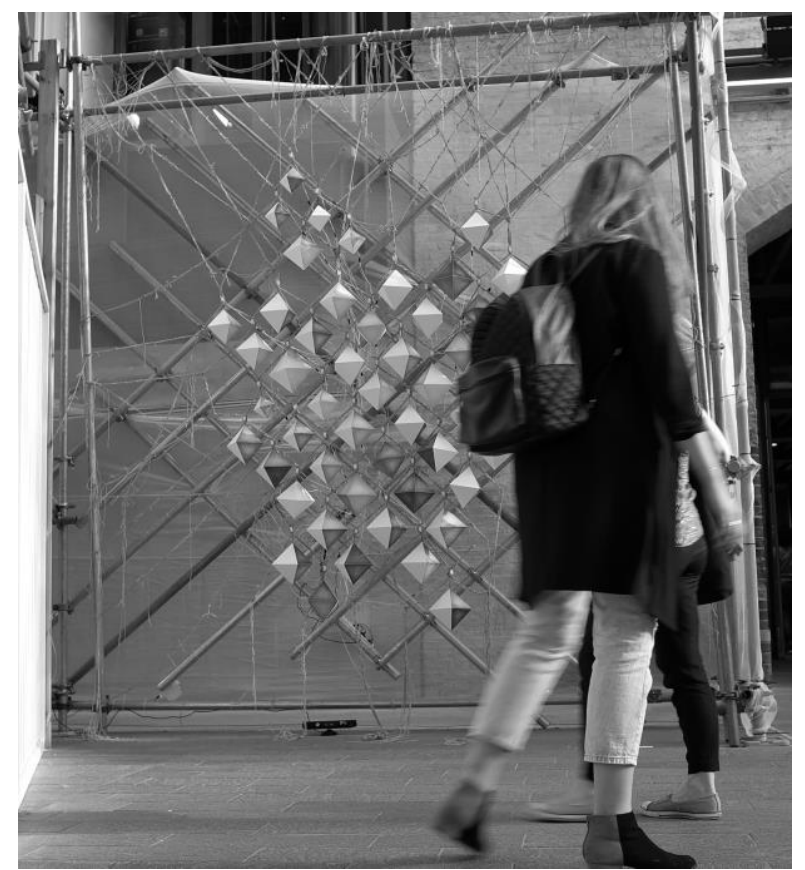

Fig. 4, People moving around in the space in front of CLOUD and remotely altering the patterns displayed, 2015. (@) Libby Heaney)

Since measurement based quantum computing was my starting point for CLOUD, it felt natural to explore complexity by promoting the viewer to the role of the measuring device/observer. In classical physics, a measurement does not irreversibly change the system's state - the disturbance is either negligible or can be determined and subtracted out. Using this distinction, the artworks $[24,25,26]$ that passively interact with the observer (i.e. the viewer does not actively change the state of the artwork) may be situated in the classical domain. On the other hand, artworks like CLOUD and Very Nervous System by David Rokeby [27], for example, that respond to the audiences input, are quantum-like as they bring the viewer into the work as a participator and the total system changes irreversibly as a result of the agential intra-actions.

\section{Intra-activity and entanglements}

The notion of intra-action was introduced by Barad [28] a component of agential realism. Agential reality is a "posthumanist performative approach to understanding technoscientific and other naturalcultural practices that specifically acknowledges and takes account of matter's dynamism." Here posthumanist refers to taking issue with anthropocentric viewpoints and a performative approach refers to direct material engagement with the world. Intra-actions (in contrast to 'interaction' "which presumes the prior existence of independent entities or relata") determine the boundary and properties of the components of phenomena and give meaning to concepts. Barad writes "The universe is agential intra-activity in its becoming" .

Agential realism draws extensively on Bohr's philosophy-physics and references quantum effects such as entanglement and quantum measurement. Entanglement and measurement were a starting point for CLOUD, so does CLOUD embody elements of Barad's theory? I believe so. The 'entanglement' of CLOUD with the participators can be seen as a series of phenomena, 
which Barad directly links to quantum physics via Bohr "For Bohr, things do not have inherently determinate boundaries or properties, and words do not have inherently determinate meanings". The phenomena (of meaning and mattering) produced by CLOUD are in continuous flux and do not have determinate boundaries or properties - they are continuously coming into being. Barad writes that "matter is produced and productive, generated and generative", which are the intraactive feedbacks between the audience and CLOUD and vice-versa -

patterns/configurations/meanings are produced and are productive of further intra-actions. By necessarily taking quantum physics as a starting point, CLOUD thus breaks down the classical object-subject, natural-cultural boundaries hence placing it and the articulated complexity firmly within the post-digital.

As a post-digital artwork, CLOUD brings together materials that are close to 'natural' (i.e. linen, paper, graphite) with digital technologies (Fig. 6) bringing to life "differential patterns of mattering" [29] between humans and non-humans. While producing the linen net, I followed a self-devised Morse code macramé (knotting) pattern (left knot $=$ dot, right knot $=$ dash) to embed undecipherable information - messages - into the net's physical texture (Fig. 5). Physicist Seth Lloyd has argued that the universe processes quantum information and this cosmic computation is responsible for the complexity of the universe, including us [30]. Lloyd's computational universe is similar to Barad's agential realism and both suggest the world is already playing the role of the contemporary artist, making real the ever-changing results (matter and meaning) of a vast computation that has been under way for billions of years. Barad writes, "Agency is not an attribute but the ongoing reconfigurations of the world." These reconfigurations are Lloyd's complex structures. Thus 'Pre-digital' and 'post-digital' are equivalent. CLOUD follows the spirit of this, revealing complexity in both the real and virtual, in nature and culture.

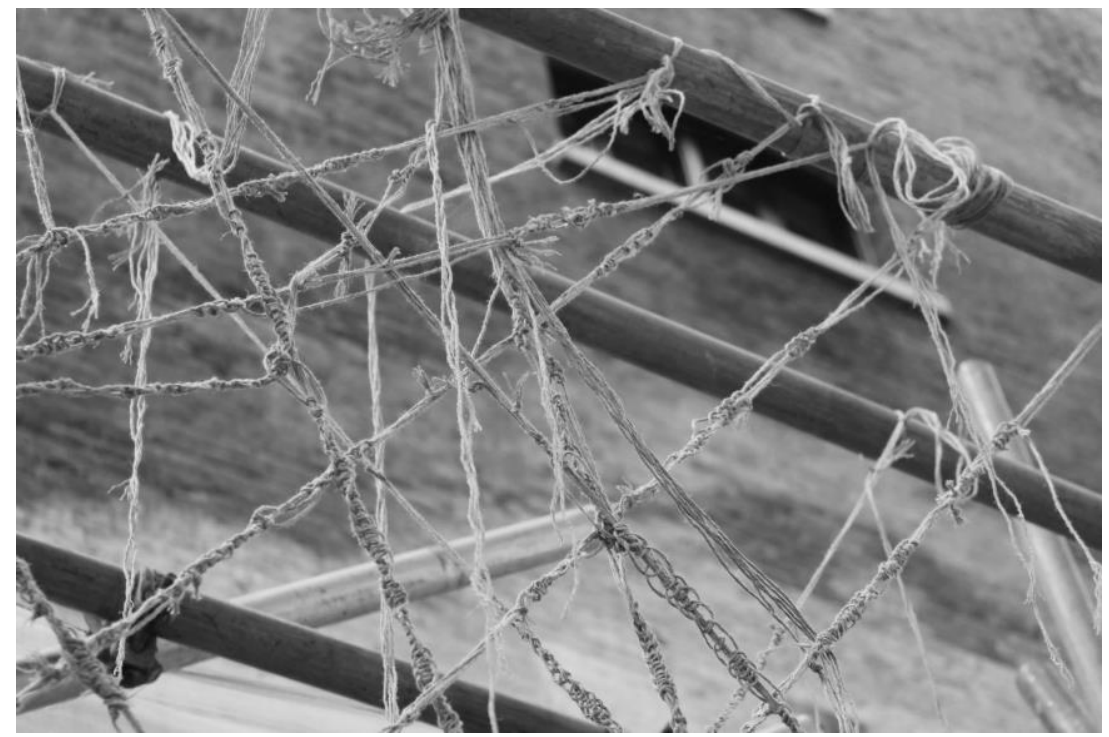

Fig. 5: The hand knotted linen net that supports the rotating pixels contains undecipherable information embedded via a Morse code like knotting macramé scheme. The metaphor of the real and virtual seems apt to explore concepts from quantum physics in the macroscopic domain. Thus the invisible sensing of the Kinect and the computer code running silently and perhaps unnoticed in the background can, I believe, be seen as metaphors of 'unpicturable' quantum processes at work. Much like how quantum computing is driven through specific material processes encoding algorithms, the audience members come to matter via these partially invisible technologies [supplementary 3]. ( Libby Heaney) 


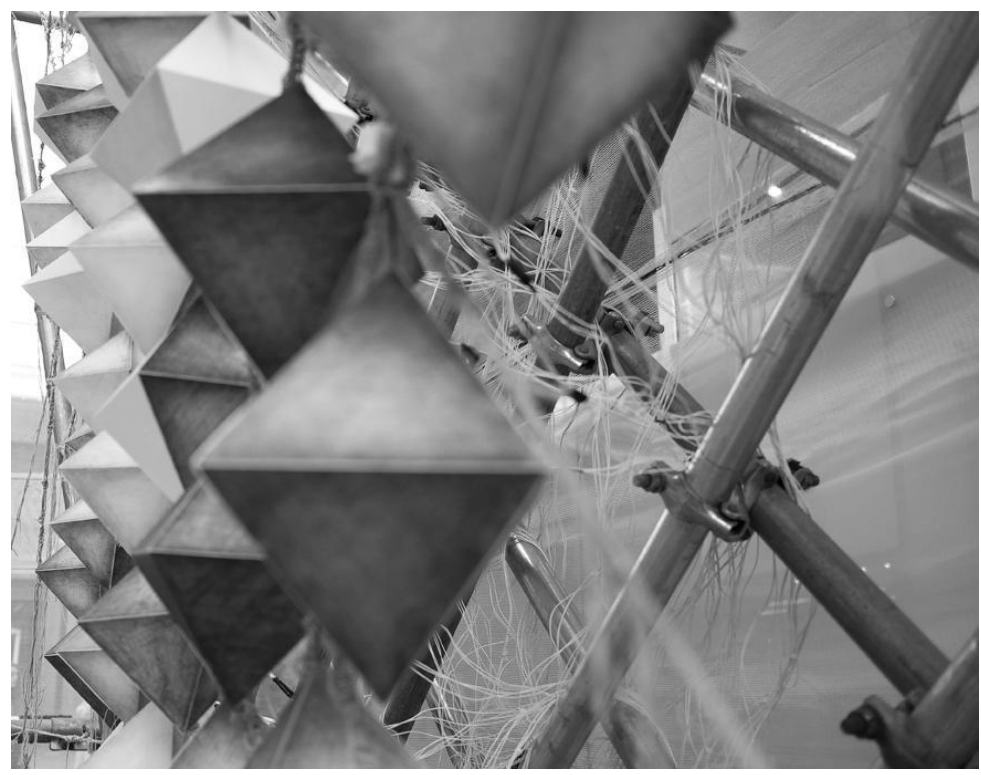

Fig. 6: The visible tangle of wires behind the pixels express a natural form and contrast the rigid geometry of the octrahedrons and scaffold. (@) Libby Heaney)

Relating art to aspects of quantum physics is not new and it is important to revisit some of these early ideas now that quantum physics has been associated with computing. Ascott wrote about a field theory for art [31] and related it to quantum physics:

"[A field theory for art] is not a one-way linearity but the interrogation of probabilities by the viewer...the viewer/observer must be a participator and is of operational importance in the total behaviour of the system. A field theory of art must pay attention to the participator."

The cluster state of measurement-based quantum computation reminds me of this field of potentiality - the potential to be projected into the outcomes of computations. CLOUD responds to this and becomes a field of probabilities incorporating the viewers. A field theory for art is intra-active art - a universe of mattering in the state of becoming. Similarly to Ascott's field theory, Barad describes how apparatuses ("the conditions of possibility for determinate boundaries and properties of objects and meanings of embodied concepts within phenomenon"[32]) "are specific material reconfigurations of the world that do not merely emerge in time but iteratively reconfigure space-timematter as part of the ongoing dynamism of becoming" [33]. Ascott also references the non-linearity of time in post-modern art. Rather than encompassing linearity, CLOUD, through it's back and forth reversible movement over time, acts as a metaphor "in its reversibility with the new physics", thereby displaying a temporal complexity as well as complexity in its spatial structure.

For Barad, concepts become specific material arrangements. CLOUD is abstracted to focus on how the interaction, form and materials articulate patterns of complexity without the complication of explicit content. Through this the audience may attribute different complex phenomena to CLOUD's structures/patterns, which themselves become the content of the work the specific material arrangements are the concepts. This may open up meanings rather than narrow them. Therefore CLOUD may be considered as meta-modern in that it re-engages 
modernist methods in order to address subject matters outside the original interest of the modernists themselves.

Taking the cluster state as inspiration, each octahedron pixel is spatially located and has an intrinsic property, like electron spin, in flux. Octahedrons are platonic solids.

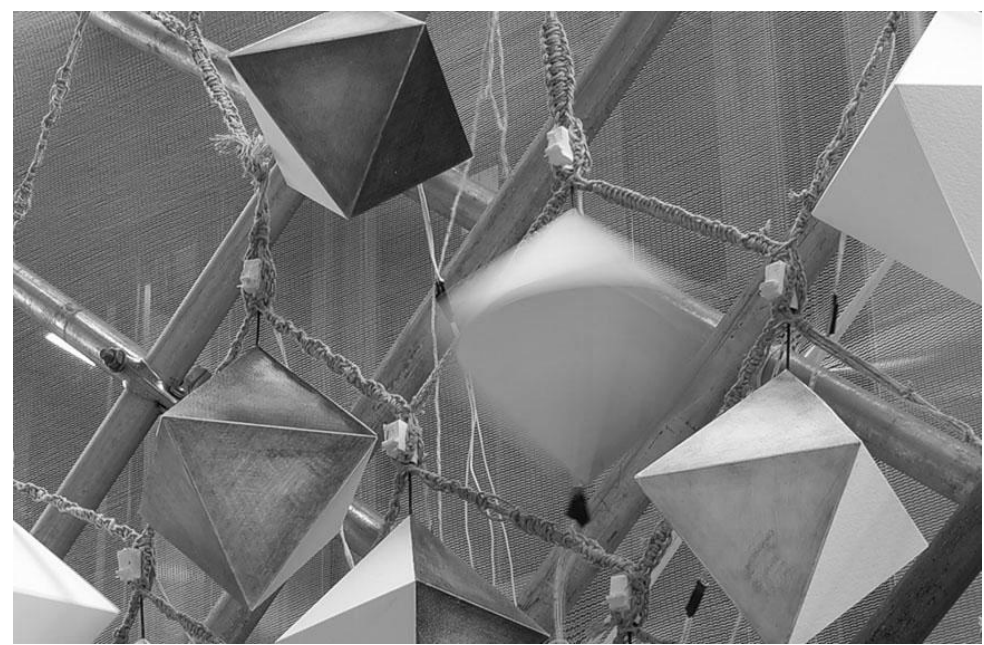

Fig. 7 the pixels in CLOUD randomly positioning, 2015. (C) Libby Heaney)

However, in CLOUD their perfect symmetry has been replaced by two distinct states - different pieces of information - the rough graphite on one side contrasting the smooth white paper on the other. This is a discontinuity in the pure platonic ideal reminiscent, but essentially a discretized (qubit) version, of wave-particle duality for our digital age. Octahedrons tessellate the space uniformly and offer up to two distinct viewpoints for audience members depending on their relative position to the left or right of the piece.

These different perspectives are a metaphor for entanglement, even for viewers outside the range of the Kinect. Barad uses the word entanglement as "not simply to be intertwined with another, as in the joining of separate entities, but to lack an independent, self-contained existence" [34]. Thus here information about the overall state of CLOUD and its meaning is delocalized across the viewers standing in the vicinity of the piece. A viewer on the left and a viewer on the right would need to come together to describe the work as a whole. For the individual viewers on the left or the right, it is as if quantum measurements has taken place, due to their mutually exclusive perspectives. While this idea of multiple perspectives is ubiquitous throughout post-modernist art (Bishop writes about it in relation to installation art, for instance [35]), by linking it to quantum entanglement new directions in expressing complexity through meaning and matter may open up with further experimentations [see supplementary material 4].

Now let us turn to the interaction of participators mediated by the Kinect. Different configurations of CLOUD and the audience continuously come into being. These intra-actions generate further intra-actions. Participators and CLOUD simultaneously become measuring apparatus and measured. Via a random number generator in the Processing code, for the same number of people in the digitally sensed area in exactly the same locations, different resulting 
patterns will occur. This is a poetic version of the measurement process in the quantum computation scheme, encouraging the participators to create structures as they explore the space (mis)understanding the algorithmic rules thereby creating further complex structures. These intra-actions give rise to Barad's phenomena where concepts are embodied in physical apparatuses and matter and meaning are entwined. As Ascott wrote [36], up close to the work, "the viewer/observer must be a participator and is of operational importance in the total behaviour of the system", they and the non-human elements are performing the computation, which like Lloyd's computational universe never reaches a final solution.

I believe the indeterminate nature of the interaction and the invisibility of the code/sensed area in front of the work encourages people to investigate and explore the complexity on their own terms - for them to experience it - through intra-activity. On the opening night of the show, I returned to the piece to find around 20 people in the sensed space jumping up and down synchronized with one another, in what I can only assume was an attempt to generate more interesting or perhaps more coherent - ordered - patterns. There were no didactic panels with details about the algorithm, hence this was an entangling of collective thought and action. This many-body entanglement of humans and non-humans, matter and meaning is an emergent phenomenon that refutes individualism.

At other times, while trying to understand the functioning of the piece, the participators would rush in from the side of the work and stand very close to the pixels (some would also wave or dance). Here I assume that by recognizing the Kinect sensor and by remembering their previous interactions with such equipment, these participators believed new patterns would be generated. In reality their presence close to the piece just randomly rotated (Fig. 7) the lowest pixels - the rest of the piece remained ordered (provided no one else was in the space). Some of these people would then stand further back and notice that pixels higher up the lattice would randomly flip, hence giving further information to the algorithmic complexity.

\section{Conclusion}

While the number of different configurations of the pixels in CLOUD cannot be infinite, the three orientations of each pixel (coloured, white or half/half) lead to $3^{54}=5.46 \times 10^{26}$ different possible patterns. Coupled with the potential of the audience, CLOUD gives rise to an infinite field theory of matter and meaning, forming a post-digital - intra-active natural and artificial computer, which similarly to Lloyd's universe uses randomness and computation to generate complexity.

\section{References and notes}

[1] Adam Curtis, Bitterlake (2015) http://www.bbc.co.uk/programmes/p02gyz6b [accessed on 23rd October 2016].

[2] Adam Curtis, Hypernormalization (2106) http://www.bbc.co.uk/programmes/p04b183c [accessed on 24th October 2016].

[3] George Marshall, Don't even think about it : Why our brains are wired to ignore climate change (Bloomsbury USA, 2015).

[4] P. W. Anderson, More is Different, Science, vol 117, pg 393 (1972). 
[5] Christos H. Papadimitriou, "Computational Complexity: A Modern Approach", (Pearson, 1993).

[6] Tor Norretranders "2011: What scientific concept would improve everybodies toolkit? Depth." https://www.edge.org/response-detail/11393 [accessed on 14th May 2016].

[7] Steven Johnson "Emergence: The connected lives of ants, brains, cities and software" (Penguin, 2001).

[8] Heather Barnett, artist website, http://heatherbarnett.co.uk/ [accessed on $8^{\text {th }}$ August 2016].

[9] Keith Tyson, artist website, http://keithtyson.com/ [accessed on 23rd October 2016].

[10] Jennifer Steinkamp, artist website, http://jsteinkamp.com/ [accessed on 23rd October 2016].

[11] University of Vienna "Shaken, not stirred: Control over complex systems consisting of many quantum particles" (2014) http://phys.org/news/2014-06-shaken-complex-quantumparticles.html [accessed on 20th April 2016].

[12] N. J. Cerf, G Leuchs, E. S. Polzik, "Quantum Information with Continuous Variables of Atoms and Light" (Singapore: World Scientific, 2007).

[13] Gross, Flammia, Eisert, "Most Quantum States Are Too Entangled To Be Useful As Computational Resources." Physical Review Letters (2009). Available online:

http://link.aps.org/doi/10.1103/PhysRevLett.102.190501. Also see http://phys.org/news/2009-05entanglement-quantum-useless.html\#jCp [accessed on $8^{\text {th }}$ August 2016].

[14] Quantum minds : Why we think like quarks

https://www.newscientist.com/article/mg21128285.900-quantum-minds-why-we-think-likequarks/ [accessed on 23rd October 2016].

[15] Karen Barad, Meeting the Universe Halfway : Quantum Physics and the Entanglement of Matter and Meaning, (Duke University Press, 2007).

[16] Arkady Plotnitsky, Complementarity (Duke University Press, 1994).

[17] James Elkins "Six stories from the end of representation" (Stanford University Press 2008).

[18] Alistair Rae, "Quantum Physics: A Beginner's Guide" (London: Oneworld Publications, 2005) and Michael Nielsen and Isaac Chuang, "Quantum Computation and Quantum Information" (Cambridge University Press, 2000).

[19] Markus Arndt, Olaf Nairz, Julian Vos-Andreae, Claudia Keller, Gerbrand van der Zouw \& Anton Zeilinger, "Wave-particle duality of C-60 molecules" Nature, vol 401, pg 680 (1999) http://www.qudev.ethz.ch/phys4/studentspresentations/waveparticle/arndt_c60molecules.pdf [accessed on 8th August 2016].

[20] Maggie McKee, "Introduction: Quantum World" New Scientist (2006) https://www.newscientist.com/article/dn9930-introduction-quantum-world/ [accessed on $8^{\text {th }}$ August 2016].

[21] There are many architectures that can are proposed as candidates for quantum computing, measurement based quantum computation is one - H. Briegel, D. E. Browne, W. Dür, R.

Raussendorf, M. Van den Nest, Nature Physics 5 1, 19-26 (2009) https://arxiv.org/abs/0910.1116 [accessed on 8th August 2016].

[22] Hans J. Briegel and Robert Raussendorf, "Persistent entanglement in arrays of interacting particles" Phys. Rev. Lett. 86, 910 (2001). 
[23] I. Bloch, "Quantum Coherence and entanglement with ultra-cold gases in optical lattices", Nature 453, 1016-1022 (19 June 2008).

http://www.nature.com/nature/journal/v453/n7198/full/nature07126.html [accessed on 20th April 2016].

[24] See Barnett [8].

[25] See Tyson [9].

[26] See Steinkamp [10].

[27] David Rokeby, "Very Nervous System", artist website

http://www.davidrokeby.com/vns.html [accessed on 23rd October 2016].

[28] See Barad [15] pg. 141.

[29] See Barad [15] pg. 140.

[30] Seth Lloyd, “The Universe As a Quantum Computer" (2013)

http://arxiv.org/pdf/1312.4455v1.pdf [accessed on 20th April 2016].

[31] Roy Ascott, "Towards a field theory for postmodernist art" printed in "Telematic Embrace: visionary theories of art, technology, and consciousness" (University of California Press:

Berkeley and Los Angeles 2003) pg 178-182.

[32] See Barad [15] pg. 143.

[33] See Barad [15] pg. 142.

[34] See Barad [15] pg. ix.

[35] Claire Bishop, "Installation Art” (London: Tate Publishing 2005).

[36] See Ascott [31] pg. 178-182.

Libby Heaney's practice and research draws primarily on science and technology both as subjects and tools. Her work is not media-specific, but typically interactive, post-digital and dealing with complexity and systems. She currently lectures at the Royal College of Art, where she runs the More is Different elective and the Systems Research Group in the School of Communications. 